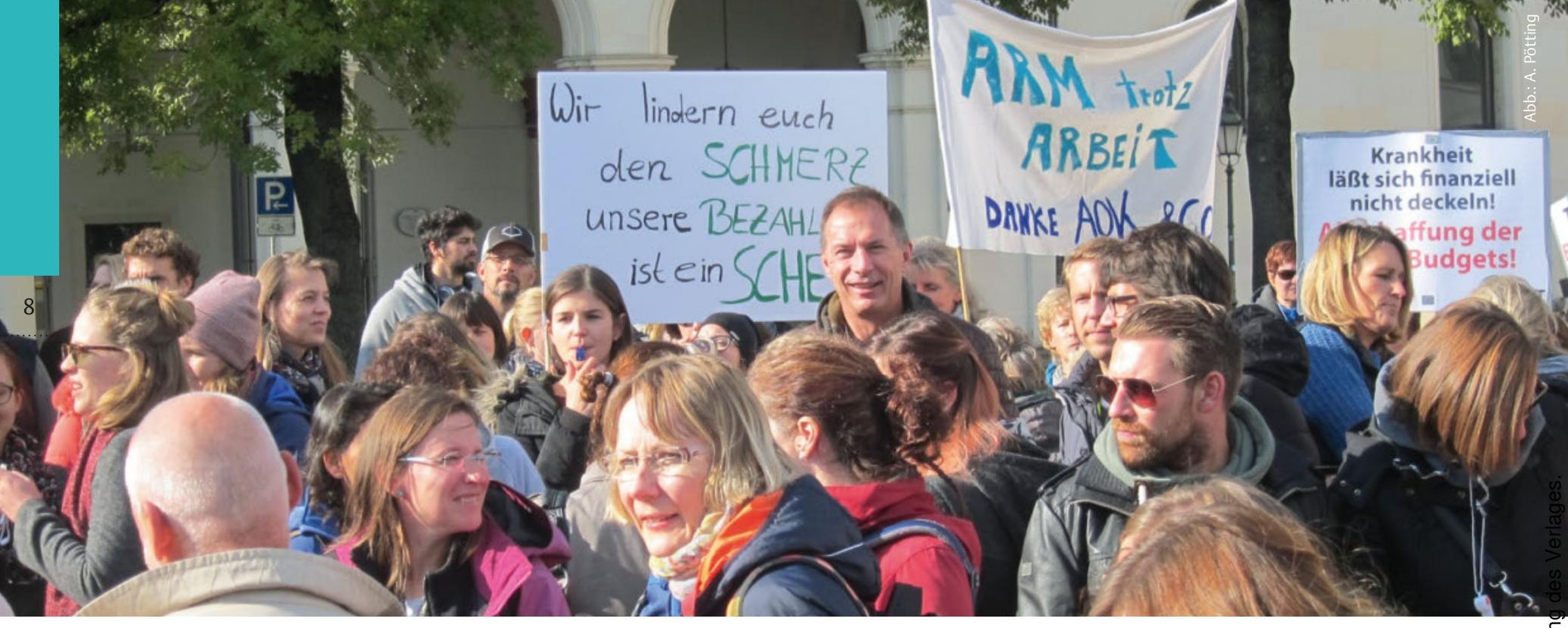

\title{
Demonstration in München
}

Bund vereinter Therapeuten kämpft weiter $\rightarrow$ Sie waren laut, sie waren wütend, doch es waren erneut zu wenig. Lediglich ein paar hundert Therapeuten kamen Samstag, den 22. Oktober 2016, nach München. Sie hatten sich aufgemacht, um für die eigene Sache zu kämpfen. Der Bund vereinter Therapeuten e. V. (BvT) hatte neben Physiotherapeuten auch Ergotherapeuten, Logopäden, Podologen und Diätassistenten geladen. Dass vor allem die großen Berufsverbände der Einladung nicht gefolgt waren, missfiel den meisten Demonstranten und war großes Diskussionsthema während des circa 2,5 Kilometer langen
Weges von der Universität über die renommierte Münchner Leopoldstraße bis hin zum Stachus. Hier am Ende der Fußgängerzone passierten tausende Menschen die Bühne des BVT, dessen Mitglieder die Chance nutzten, um die Passanten direkt anzusprechen und Flyer zu verteilen. Die Therapeuten waren gekommen, um für bessere Bedingungen in den Therapieberufen zu kämpfen.

Im nächsten Jahr will der BVT am 17. Juni 2017 erneut in Berlin demonstrieren. Sie erhoffen sich aufgrund der Bundestagswahl mehr Interesse von Seiten der Politiker und auch der Therapeuten.

\section{Mehr Geld für Heilmittel}

Rahmenvorgaben für 2017 vereinbart

$\rightarrow$ Im Herbst jeden Jahres legen die Kassenärztliche Bundesvereinigung (KBV) und der GKV-Spitzenverband fest, in welcher Höhe sie die Heilmittelausgaben für das kommende Jahr erwarten. Diese Rahmenvorgabe ist Richtschnur für die Verhandlungen der Heilmittelrichtgrößen auf Länderebene zwischen Kassenärztlicher Vereinigung und Krankenkassen. Bei den Heilmitteln gehen die beiden Vereinigungen davon aus, dass diese 2017 um 4,9 Prozent (etwa 280 Millionen Euro) steigen werden, und begründen das mit den neuen Regelungen zu den besonderen Verordnungsbedarfen (Praxisbesonderheiten) und langfristigen Heilmittelbedarfen (Langfristverordnungen), die zum 1. Januar 2017 in Kraft getreten sind. Deren Diagnoselisten wurden erweitert. So gibt es mehr Indikationen, für die

Patienten langfristig Ergotherapie verordnet bekommen können.

Ob sich das auf das Verordnungsverhalten der Ärzte auswirkt, wird sich zeigen. Der SWR berichtete Anfang Dezember, dass 2016 in Baden-Württemberg die Ärzte aus Angst vor Strafzahlungen deutlich weniger Ergo- und Physiotherapie verordnet hatten. ba

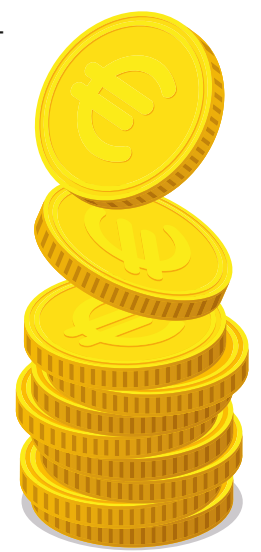




\section{Pflegestufen werden zu Pflegegraden}

Neu ab Januar $2017 \rightarrow$ Durch das zweite Pflegestärkungsgesetz, das am 1. Januar 2016 in Kraft trat, wurden die Weichen für einen neuen Pflegebedürftigkeitsbegriff gestellt: Zum 1. Januar 2017 wurden die drei Pflegestufen durch fünf neue Pflegegrade ersetzt. Bisher wurde zwischen Pflegebedürftigen mit körperlichen Einschränkungen sowie Pflegebedürftigen mit kognitiven und psychischen Einschränkungen unterschieden. Diese Differenzierung soll künftig wegfallen. Entscheidend sollen allein die persönlichen Beeinträchtigungen und Fähigkeiten jedes Einzelnen sein: Was kann er noch alleine und wobei benötigt er Unterstützung? Das soll den individuellen Bedürfnissen Pflegebedürftiger besser gerecht werden, vor allem Menschen mit Demenz. Die Unterstützung setzt deutlich früher an, so werden bei- spielsweise in Pflegegrad 1 Menschen eingestuft, die noch keine erheblichen Beeinträchtigungen haben, aber schon in gewissem Maß - zumeist körperlich eingeschränkt sind. Bei schwerster Beeinträchtigung der Selbstständigkeit, die eine besondere pflegerische Versorgung erfordert, gilt Pflegegrad 5.

Werden Betroffene von Angehörigen gepflegt, können sie Pflegegeld in Anspruch nehmen, das sie auch mit Leistungen der amubulanten Pflege kombinieren können. Zudem erhalten Pflegebedürftige aller Pflegegrade, die im häuslichen Umfeld gepflegt werden, einen zweckgebundenen Entlastungsbetrag. Dieser soll beispielsweise bei der hauswirtschaftlichen Versorgung unterstützen. ba

$\boldsymbol{\theta}$ www.pflegestaerkungsgesetz.de

\section{Modellvorhaben von zehn auf vier Jahre verkürzt}

Drittes Pflegestärkungsgesetz beschlossen $\rightarrow$ Am 1. Dezember 2016 hat der Deutsche Bundestag das dritte Pflegestärkungsgesetz verabschiedet, das auch für die Therapieberufe bedeutende Neuerungen mit sich bringt: Die Modellphase der primärqualifizierenden Studiengänge in der Physiotherapie, Ergotherapie, Logopädie und Hebammenkunde wird nur auf vier Jahre verlängert. Das Bundesgesundheitsministerium (BMG) wollte sie ursprünglich auf weitere zehn Jahre ausdehnen.

Seit 2009 können die Bundesländer für die Ausbildung von Ergotherapeuten, Logo- päden, Physiotherapeuten und Hebammen aufgrund der Einführung einer Modellklausel in die Berufsgesetze ein grundständiges Studium an Hochschulen erproben. Zum 31. Dezember 2017 wäre die Modellphase ausgelaufen. Als im August 2016 das BMG für eine Verlängerung um zehn Jahre plädierte, hagelte es von den Berufsverbänden, Hochschulen und manchen Politikern, beispielsweise dem Bundestagsabgeordneten Dr. Roy Kühne, Protest. Mit Erfolg.

Bleibt zu hoffen, dass dann zum 1. Januar 2022 die Modellstudiengänge in Regelstudiengänge überführt werden können.

\section{Vorsicht vor Einzelverträgen mit den Kassen}

AOK Sachsen-Anhalt $\rightarrow$ In SachsenAnhalt hat die AOK bei manchen Praxisinhabern überraschend die Abrechnungen gekürzt. Die Begründung: Die Inhaber hätten die neuen Verträge nicht unterschrieben, deshalb gelten die von DVE und AOK Sachsen-Anhalt neu verhandelten Preise nicht. ergopraxis ging dieser Sache nach.

In Sachsen-Anhalt verschickte die AOK Individualverträge an Praxisinhaber. Sie hatten die Wahl zwischen einem neuen Vertrag, der ein schwer durchschaubares Preisangebot enthielt, oder einer sogenannten Anerkenntniserklärung des Rahmenvertrags. Wer nicht darauf reagierte und kein DVE-Mitglied ist, profitiert nun nicht von den mittlerweile zwischen DVE und AOK Sachsen-Anhalt ausgehandelten Preiserhöhungen von im Schnitt 15 Prozent statt 8 Prozent.

Yvonne Görmar, Referentin für Praxisangelegenheiten beim DVE, kennt die Problematik der Einzelverträge seit circa anderthalb Jahren. Grundsätzlich sind nach § 125 Abs. 2 SGB V individuelle Verträge zwischen Praxisinhabern und allen Krankenkassen möglich, bestätigt Prof. Dr. Dr. Thomas Ufer, Arzt und Fachanwalt für Medizinrecht. Möglicherweise könnte eine bewusste Spaltung der Leistungserbringer das Ziel der Krankenkasse sein, da die Verhandlungsposition einzelner Therapeuten wesentlich schlechter ist als die des Berufsverbandes. Dessen Position würde durch viele Einzelverträge sinken.

Thomas Ufer und Yvonne Görmar raten davon ab, individuelle Verträge mit den Kassen zu unterzeichnen. Die geschilderten Rechnungskürzungen scheinen juristisch möglich, wenn die im betreffenden Bundesland niedergelassenen Therapeutinnen weder den individuellen Vertrag noch die Anerkenntniserklärung des Rahmenvertrags unterzeichnet haben.

Michael Schiewack 